\title{
Escitalopram and paroxetine in the treatment of generalised anxiety disorder
}

\author{
Randomised, placebo-controlled, double-blind study*
}

DAVID S. BALDWIN, ANNA KARINA TRAP HUUSOM and ELI MAHLUM

\begin{abstract}
Background It is uncertain whether higher doses of selective serotonin reuptake inhibitors have greater efficacy in generalised anxiety disorder.
\end{abstract}

\begin{abstract}
Aims To assess the efficacy of different doses of escitalopram in generalised anxiety disorder.
\end{abstract}

Method Randomised, double-blind, placebo-controlled, fixed-dose, parallelgroup, 12-week study, with 681 patients: placebo $(n=139)$; escitalopram, $5 \mathrm{mg} /$ day, $(n=134) ; 10 \mathrm{mg} /$ day $(n=136) ; 20 \mathrm{mg} /$ day $(n=133)$; paroxetine, $20 \mathrm{mg} /$ day $(n=139)$.

Results Mean change in the primary efficacy measure was greater with escitalopram 10 and $20 \mathrm{mg}$ than with placebo; $10 \mathrm{mg}$ was more efficacious than paroxetine. Paroxetine was superior to placebo on some secondary measures, at some time points. Compared with placebo, more patients withdrew because of adverse events with escitalopram $20 \mathrm{mg}$ and paroxetine.

\section{Conclusions Escitalopram was} efficacious in generalised anxiety disorder, 20 was not significantly superior to $10 \mathrm{mg} /$ day. Escitalopram $10 \mathrm{mg}$ was more efficacious than paroxetine.

\section{Declaration of interest The University of Southampton has received support from Lundbeck and GlaxoSmith Kline. D.S.B. has received consultancy honoraria from Lundbeck and GlaxoSmith Kline. A.K.T.H. and E.M. are employees of $\mathrm{H}$. Lundbeck A/S, who sponsored this study.}

Some selective serotonin reuptake inhibitors (SSRIs) and benzodiazepines, and the serotonin-noradrenaline reuptake inhibitor venlafaxine, are efficacious in generalised anxiety disorder (Baldwin \& Polkinghorn, 2005). Placebo-controlled double-blind studies demonstrate the efficacy of the SSRIs paroxetine (Pollack et al, 2001; Rickels et al, 2003), sertraline (Allgulander et al, 2004; Brawman-Mintzer et al, 2006), and escitalopram (Davidson et al, 2004; Goodman et al, 2005). A 24-week study found escitalopram (10-20 mg/day) and paroxetine $(20-50 \mathrm{mg} /$ day $)$ to have similar efficacy (Bielski et al, 2005). These studies provide no clear evidence for a dose-response relationship. Current guidelines for assessing efficacy recommend a minimum length of 8 weeks, using a placebocontrolled design comprising fixed doses to establish optimal dosage (Montgomery $\&$ van Zwieten-Boot, 2002). This study aimed to compare the efficacy of fixed doses of escitalopram $(5,10$ or $20 \mathrm{mg} /$ day $)$ with placebo over 12 weeks' treatment, including paroxetine $(20 \mathrm{mg} /$ day $)$ as an active reference.

\section{METHOD}

\section{Study design and closing schedule}

This randomised, placebo-controlled, fixeddose, active-reference study included 63 centres in 10 countries. It was conducted in accordance with the principles of Good Clinical Practice (ICH, 1996) and the Declaration of Helsinki (World Medical Association, 2000). Local ethics committees approved the study design, and eligible patients gave their written informed consent before participating. After screening,

\footnotetext{
* Some of the results in this manuscript were presented as a poster at the 17th Congress of the European College of Neuropsychopharmacology, Stockholm, Sweden, 9-13 October 2004
}

patients entered a 1-week, single-blind, placebo lead-in period before being randomised to 12 weeks of double-blind treatment with fixed doses of escitalopram $(5,10$ or $20 \mathrm{mg} /$ day $)$, paroxetine $(20 \mathrm{mg} /$ day), or placebo. Patients who completed double-blind treatment entered a randomised staggered 2-week (1-week doubleblind, then 1-week single-blind) placebo wash-out period. Efficacy and tolerability were assessed at baseline and after 1, 2, 4, $6,8,10,12,13$ and 14 weeks; a safety follow-up visit was performed 14 days after the last wash-out visit.

\section{Allocation to treatment}

Study medications were capsules for oral administration, of identical appearance, taste and smell. The oxalate salt of escitalopram was used in the capsules. Patients who met selection criteria at the screening and baseline visits were assigned to 12 weeks of double-blind treatment in a 1:1:1:1:1 ratio of $5 \mathrm{mg}$ escitalopram to $10 \mathrm{mg}$ escitalopram to $20 \mathrm{mg}$ escitalopram to $20 \mathrm{mg}$ paroxetine to placebo according to a computer-generated randomisation list drawn up by $\mathrm{H}$. Lundbeck $\mathrm{A} / \mathrm{S}$. The timing of down-titration for the $5 \mathrm{mg}$ and $10 \mathrm{mg}$ escitalopram groups and the $20 \mathrm{mg}$ paroxetine group was built into the overall randomisation scheme; patients in these groups were assigned to continue current active treatment or start placebo wash-out treatment at week 13 in a 1:1 ratio of active treatment to placebo. The details of the randomisation series were unknown to any of the investigators and were contained in a set of sealed opaque envelopes. At each study centre, sequentially enrolled patients were assigned the lowest randomisation number available in blocks of ten. All study personnel and participants were masked to treatment assignment for the duration of the study.

\section{Patient population}

The selection criteria were chosen to select physically healthy male and female out-patients with a primary diagnosis of generalised anxiety disorder according to DSM-IV-TR (American Psychiatric Association, 2000) criteria. The Mini International Neuropsychiatric Interview (MINI: Sheehan et al, 1998) was used to establish the diagnosis and to confirm the 
presence or absence of other disorders. Patients aged 18-65 years old with a Hamilton Anxiety Scale (HAMA; Hamilton, 1959) total score $\geqslant 20$, and a score of $\geqslant 2$ on both HAMA item 1 (anxious mood) and item 2 (tension) at screening and at baseline could be included. A low level of depressive symptoms was allowed using the Montgomery-Åsberg Depression Rating Scale (MADRS; Montgomery \& Åsberg, 1979 ), i.e. total score $\leqslant 16$ at screening and at baseline.

Patients with the following disorders within the previous 6 months (based on DSM-IV-TR criteria, confirmed using the MINI) were excluded: major depressive disorder, panic disorder, social anxiety disorder, post-traumatic stress disorder, bipolar disorder, obsessive-compulsive disorder, eating disorders, body dysmorphic disorder, substance misuse disorder, any personality disorder that could jeopardise the evaluation of the treatment for primary generalised anxiety, as judged by the investigator, and any current or previous psychotic disorder as defined by DSM-IVTR. Patients were also excluded if they were at risk of suicide (according to the investigator's judgement), had a score $>3$ on item 10 (suicidal thoughts) of the MADRS, or had made a serious suicide attempt within the past year or were receiving cognitive-behavioural therapy, electroconvulsive therapy, cognitive therapy or problem-solving treatment, or planned to initiate such therapy. Furthermore, patients with an unstable serious illness and/or serious sequelae of liver or renal insufficiency, or cardiac, vascular, pulmonary, gastrointestinal, endocrine, neurological, infectious, neoplastic or metabolic disturbance were also excluded. Patients were excluded if they had taken psychoactive substances, anxiolytics, antidepressants, monoamine oxidase inhibitors, benzodiazepines, $\beta$-blockers (use of anti-hypertensives other than $\beta$-blockers was permitted as long as the dose had been stable for 6 months and remained fixed during the study), tryptophan, oral antipsychotics, narcotic analgesics (except intermittent use of codeine-based analgesics), warfarin sodium, digitalis, cardiac glycosides, type $1 \mathrm{c}$ antiarrhythmics, phenytoin, cimetidine, regular daily therapy with any hypnotic (except zolpidem, zopiclone, or zaleplon for insomnia, but not more than 3 times per week), psychoactive herbal remedies, antiepileptics, ongoing prophylactic treatment with lithium, valproate or carbamazepine, and triptans within the 2 weeks before the screening visit, and any investigational drug or depot antipsychotics within 6 months before the screening visit.

\section{Efficacy assessments}

The primary end-point was defined as the adjusted mean change in HAMA total score from baseline to week 12, based on the intention-to-treat set and using last-observation-carried-forward analysis.

Secondary efficacy measures included: change from baseline in HAMA total score at each visit; Clinical Global Impression Severity (CGI-S) and Clinical Global Impression - Improvement (CGI-I; Guy, 1976) score per visit; proportion of responders per visit using two criteria $(\geqslant 50 \%$ reduction in HAMA total score compared with baseline, and CGI-I score of 1 and 2 ); proportion of remitters (HAMA total score $\leqslant 7)$ per visit; and change from baseline in the self-rating Hospital Anxiety and Depression Scale (HAD; Zigmond \& Snaith, 1983) anxiety sub-scale score at weeks 6 and 12. The investigators were trained by a physician experienced in the use of HAMA before inclusion of patients into the study to increase interrater reliability. Patient ratings were conducted by the same person at each visit, whenever possible.

\section{Tolerability assessments}

Tolerability was based on the incidence of adverse events throughout the study. The Discontinuation Emergent Signs and Symptoms (DESS) scale is a 43-item checklist (Rosenbaum et al, 1998) designed to assess possible treatment-related discontinuation symptoms. For this study, the DESS was slightly modified to include four extra items, reported after stopping SSRI treatment: vivid dreams, electric shock-like sensations, somnolence, and feeling tense. An event was considered discontinuation emergent if it appeared during the previous 7 days, or if a previously reported event had worsened. The modified DESS was assessed at week 12 and during the wash-out period (weeks 13 and 14) for patients who had completed the 12-week double-blind treatment period. Unresolved symptoms in the DESS checklist were subject to enquiry at the safety follow-up visit. Half of the patients randomised to escitalopram
5 or $10 \mathrm{mg} /$ day, or $20 \mathrm{mg} /$ day paroxetine, received placebo during the 2 -week washout period, whereas the other half continued on active treatment for 1 week (week 13) and received placebo for the second week (week 14). Patients who were randomised to $20 \mathrm{mg}$ escitalopram were down-titrated to $10 \mathrm{mg}$ escitalopram for 1 week (week 13) before they received placebo (week 14).

\section{Statistical analysis}

A minimum of 130 patients in each treatment group (intention-to-treat) was expected to provide a standardised effect size of 0.35 , that is a significant treatment difference from placebo of at least $35 \%$ of the pooled standard deviation when comparing the mean change from baseline to week 12 (last observation carried forward) in HAMA total score, using a two-sided $t$-test with $80 \%$ power at a $5 \%$ level of significance.

All efficacy analyses were conducted on the intention-to-treat population consisting of all randomised patients who took at least one dose of double-blind study medication and who had at least one valid postbaseline assessment of the HAMA. The prospectively defined primary efficacy end-point was the adjusted mean change from baseline in HAMA total score at week 12 , based on intention to treat (last observation carried forward). Comparisons of the primary efficacy end-point between escitalopram and placebo were made using analysis of covariance (ANCOVA) with treatment and centre as fixed factors, and with the baseline HAMA total score as a covariate. To adjust for multiple testing, an $F$-test was used to test the overall null hypothesis of equal mean changes in the three escitalopram groups and the placebo group. If the overall $F$-test was significant at the $5 \%$ level, pairwise comparisons of each of the three escitalopram dose groups and the placebo group were made using two-sided $t$-tests with the overall mean square error as the error term at a $5 \%$ level of significance. Likewise, paroxetine was compared pairwise with the other treatment groups using two-sided $t$-tests with the overall mean square error as the error term at a $5 \%$ level of significance.

The secondary efficacy analyses of mean change from baseline to each visit in the HAMA total scores and HAD sub-scale score were analysed by ANCOVA (observed cases and last observation carried 
forward) using the model described for the primary analysis. The CGI-I scores were analysed using analysis of variance. Between-group comparisons of patients considered to be treatment responders and between patients considered to be remitters were carried out using Fisher's exact test.

Incidences of adverse events were compared between treatment groups using Fisher's exact test based on all randomised patients who took at least one dose of double-blind medication.

The modified DESS total scores during the wash-out period were analysed for patients completing the study based on observed cases using ANCOVA with treatment and centre as factors, and the modified DESS total score at the start of the wash-out period as a covariate.

\section{RESULTS}

\section{Patient baseline characteristics}

There were no clinically relevant differences between groups in patient demographic or clinical characteristics at baseline (Table 1). The small differences between groups in HAMA total score at baseline are unlikely to be of clinical significance, and are adjusted for in the primary efficacy analysis by the inclusion of baseline score as a covariate. Most patients were Caucasian, and there was an approximately 2:1 ratio of women to men, with a mean age of about 41 years. Baseline HAMA, CGI-S and MADRS scores indicated a moderately to severely ill patient population with a low level of depressive symptoms.

\section{Withdrawals from the study}

Fig. 1 shows the patient disposition for the 14-week study period for all groups. A total of 98 patients $(14 \%)$ withdrew from the study during the 12-week, double-blind period (Table 2), and withdrawal rates ranged from $10.8 \%$ to $18.7 \%$.

The proportion of patients that withdrew because of adverse events was relatively low $(<11 \%$ in any treatment group and $<7 \%$ overall). Compared with the placebo group, significantly more patients (chi-square test, $P<0.05$ ) in the escitalopram $20 \mathrm{mg}$ and paroxetine $20 \mathrm{mg}$ groups withdrew because of adverse events. Withdrawal rates due to lack of efficacy in the escitalopram 5 and $20 \mathrm{mg}$, paroxetine $20 \mathrm{mg}$ and placebo groups were comparable.
Table I Baseline patient characteristics

\begin{tabular}{|c|c|c|c|c|c|}
\hline & PBO & ESC 5 mg & $\mathrm{ESC} 10 \mathrm{mg}$ & ESC $20 \mathrm{mg}$ & PAR $20 \mathrm{mg}$ \\
\hline Patients randomised, $n^{\prime}$ & 139 & 134 & 136 & 133 & 140 \\
\hline Patients treated, $n$ & 139 & 134 & 136 & 133 & 139 \\
\hline Women, $n$ (\%) & $93(67)$ & $78(58)$ & $91(67)$ & $92(69)$ & $84(60)$ \\
\hline \multicolumn{6}{|l|}{ Age, years } \\
\hline Mean (s.d.) & $41.8(I 1.6)$ & 40.7 (II.9) & $41.8(12.8)$ & $41.0(12.2)$ & $41.7(12.0)$ \\
\hline Range & $19-64$ & $18-65$ & $19-65$ & $19-65$ & $18-64$ \\
\hline Caucasian, $n(\%)$ & $138(99.3)$ & $132(98.5)$ & $135(99.3)$ & |3| (98.5) & I37 (98.6) \\
\hline \multicolumn{6}{|l|}{ Efficacy scores ${ }^{2}$} \\
\hline HAMA total score (s.d.) & $27.1(4.6)$ & $27.1(4.5)$ & $26.0(4.1)$ & $27.7(4.9)$ & $27.3(4.2)$ \\
\hline HAD anxiety sub-scale score (s.d.) & $13.5(3.6)$ & I3.1 (3.4) & I3.I (3.7) & I3.6(3.3) & $13.0(3.0)$ \\
\hline CGI-S (s.d.) & $4.6(0.7)$ & $4.6(0.8)$ & $4.5(0.7)$ & $4.6(0.7)$ & $4.6(0.7)$ \\
\hline MADRS total score (s.d.) & II.4 (3.2) & II.2(3.0) & II.0(3.I) & II.4(3.0) & $11.0(3.1)$ \\
\hline
\end{tabular}

CGI-S, Clinical Global Impression - Severity; ESC, escitalopram; HAD, Hospital Anxiety and Depression Scale; HAMA, Hamilton Rating Scale for Anxiety; MADRS, Montgomery-Åsberg Depression Rating Scale; PAR, paroxetine; PBO, placebo.

I. Randomised patients per country: Czech Republic, 62; Denmark, 75; Estonia, 84; Finland, 107; France, 44; Germany, 67. Holland, 4; Norway, 54: Spain, 44; UK, I4I; total, 682

2. Based on intention-to-treat population (see Fig. I)
Compared with placebo, significantly fewer patients in the escitalopram $10 \mathrm{mg}$ group withdrew because of lack of efficacy.

\section{Primary efficacy analysis}

The prospectively defined primary efficacy end-point (adjusted mean change in HAMA total score from baseline to week 12 , last observation carried forward) showed that treatment with escitalopram 10 and $20 \mathrm{mg}$ was significantly superior to placebo at week 12 (Table 3 and Fig. 2). Escitalopram $5 \mathrm{mg}$ and paroxetine $20 \mathrm{mg}$ were not significantly superior to placebo at week 12.

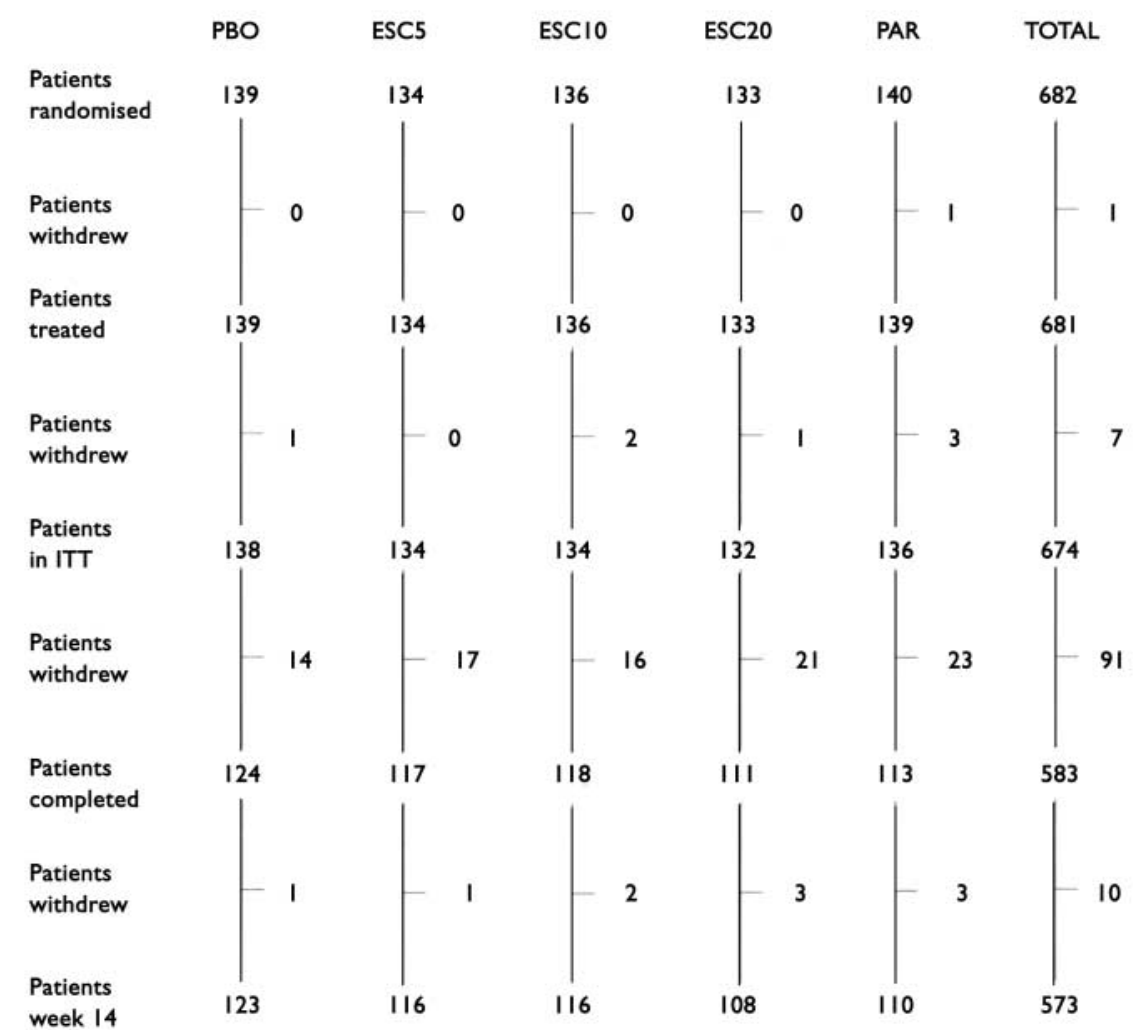

Fig. I Patient disposition for the I4-week study period. ESC, escitalopram (5, 10 or $20 \mathrm{mg}$ ); ITT, intention-totreat; PAR, paroxetine; PBO, placebo. 
Table 2 Withdrawals from study according to primary reason during the 12-week study period

\begin{tabular}{|c|c|c|c|c|c|}
\hline & PBO & ESC $5 \mathrm{mg}$ & $\mathrm{ESC} 10 \mathrm{mg}$ & ESC $20 \mathrm{mg}$ & PAR $20 \mathrm{mg}$ \\
\hline Patients randomised, $n$ & 139 & 134 & 136 & 133 & 140 \\
\hline Patients treated, $n$ & 139 & 134 & 136 & 133 & 139 \\
\hline Patients withdrawn, $n$ (\%) & $15(10.8)$ & $17(12.7)$ & $18(13.2)$ & $22(16.5)$ & $26(18.7)$ \\
\hline \multicolumn{6}{|l|}{ Primary reason, $n$ (\%) } \\
\hline Adverse event(s) & $4 \quad(2.9)$ & $7(5.2)$ & 8 (5.9) & $14(10.5)^{*}$ & $13(9.4)^{*}$ \\
\hline Lack of efficacy & $5(3.6)$ & $5(3.7)$ & $0(0)^{*}$ & $2(1.5)$ & $4(2.9)$ \\
\hline Withdrawal of consent & $4(2.9)$ & $0(0)$ & I (0.7) & I $(0.8)$ & $3(2.2)$ \\
\hline Non-compliance & I (0.7) & I $(0.7)$ & $2(1.5)$ & I $(0.8)$ & $0(0)$ \\
\hline Protocol violation & I $(0.7)$ & I (0.7) & $5(3.7)$ & $3(2.3)$ & $4(2.9)$ \\
\hline Administrative or other & $0(0)$ & $2(1.5)$ & $0(0)$ & I $(0.8)$ & I (0.7) \\
\hline Lost to follow-up & $0(0)$ & I (0.7) & $2(1.5)$ & $0(0)$ & I $(0.7)$ \\
\hline
\end{tabular}

ESC, escitalopram; PAR, paroxetine; PBO, placebo.

$* P<0.05$ v. placebo $\left(\chi^{2}\right.$-test).

Table 3 Mean change from baseline to week 12 in HAMA total score (ITT, LOCF)

\begin{tabular}{|c|c|c|c|c|c|c|}
\hline & \multicolumn{3}{|c|}{ Treatment } & \multicolumn{3}{|c|}{ Difference v. placebo' } \\
\hline & $n$ & Mean change & s.e. & Difference & $95 \% \mathrm{Cl}$ & $P$ \\
\hline PBO & 138 & -14.20 & 0.66 & & & \\
\hline ESC 5 mg & 134 & -15.49 & 0.67 & -1.29 & -3.13 to 0.54 & 0.165 \\
\hline ESC $10 \mathrm{mg}$ & 134 & -16.76 & 0.68 & -2.56 & -4.40 to -0.73 & 0.006 \\
\hline ESC $20 \mathrm{mg}$ & 132 & -16.35 & 0.68 & -2.15 & -3.99 to -0.31 & 0.022 \\
\hline PAR $20 \mathrm{mg}$ & 136 & -14.71 & 0.67 & -0.51 & -2.33 to $\mathrm{I} .32$ & 0.585 \\
\hline
\end{tabular}

ESC, escitalopram; ITT, intention-to-treat; LOCF, last observation carried forward; PAR, paroxetine; PBO, placebo I. Analysis of covariance.

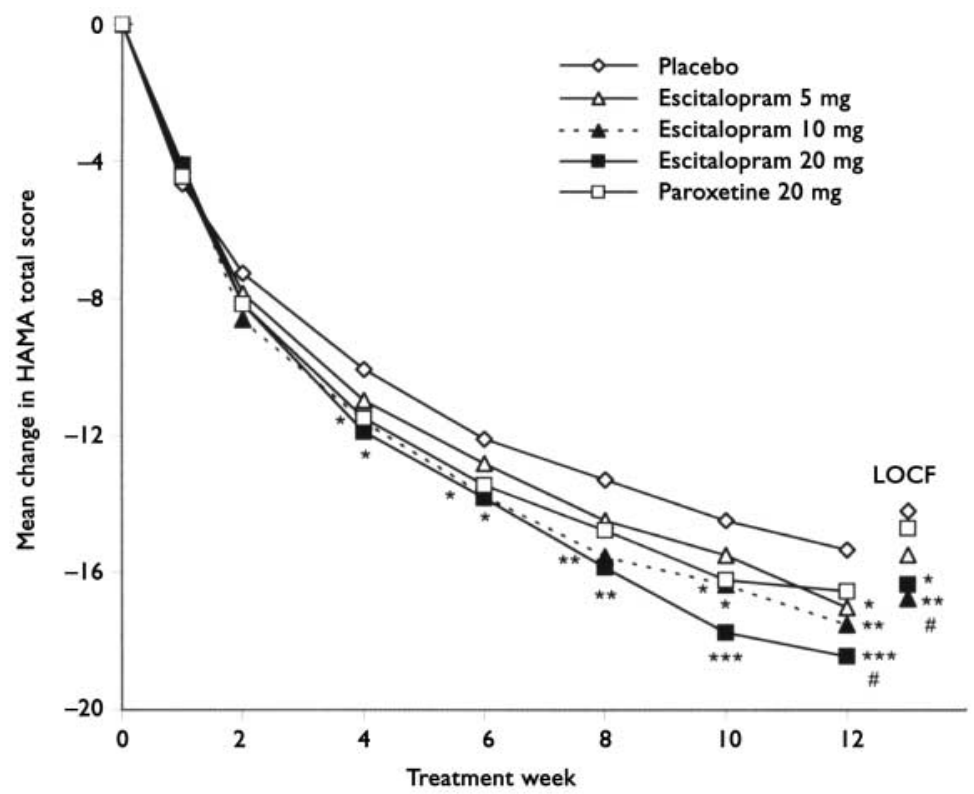

Fig. 2 Mean change from baseline in Hamilton Rating Scale for Anxiety (HAMA) total scores by visit (intention-to-treat, observed cases) and at week 12, LOCF (last observation carried forward). Difference $v$. placebo, $* P<0.05$; **P<0.01; ${ }^{* * * P}<0.001$. Difference $v$. paroxetine ${ }^{\# P}<0.05$ (analysis of covariance).
The mean HAMA total scores decreased in all treatment groups from approximately 27 at baseline to less than 13 at week 12 . The last-observation-carried-forward analysis demonstrated that escitalopram $10 \mathrm{mg}$ was also significantly (ANCOVA, $P<0.05$ ) superior to paroxetine $20 \mathrm{mg}$ at week 12 (HAMA difference of -2.06 ; $95 \%$ CI -3.90 to -0.21 ). In observed cases analyses, all three doses of escitalopram were significantly more efficacious than placebo at week 12 (ANCOVA, $P<0.05)$, and showed a clear dose-response relationship (difference from placebo; escitalopram $5 \mathrm{mg}$ : $-1.67(95 \% \mathrm{CI}$ -3.25 to $-0.09 ; P<0.05)$, escitalopram $10 \mathrm{mg}$ : $-2.17(95 \% \mathrm{CI}-3.75$ to -0.59 ; $P<0.01)$, escitalopram $20 \mathrm{mg}:-3.10$ (95\% CI -4.72 to $-1.49 ; P<0.001$ ), Fig. 2). Separation of active treatment from placebo was apparent from week 4 onwards for escitalopram $10 \mathrm{mg}$ and $20 \mathrm{mg}$ (ANCOVA, $P<0.05$ ). A statistically significant separation of paroxetine $20 \mathrm{mg}$ from placebo was seen at week 10 (ANCOVA, $P<0.05)$. Analysis revealed that escitalopram $20 \mathrm{mg}$ was significantly (ANCOVA, $P<0.05)$ superior to paroxetine $20 \mathrm{mg}$ at week 12 (observed cases) (HAMA difference of $-1.90 ; 95 \%$ CI -3.54 to -0.25 ).

\section{Secondary efficacy analysis}

CGI-Improvement scores

The mean CGI-I scores at each visit are shown in Fig. 3. In the observed cases analyses, separation of active treatment from placebo was statistically significant from week 2 onwards for escitalopram 10 and $20 \mathrm{mg}$ (ANOVA, $P<0.05$ ), including week 12 (last observation carried forward). Escitalopram $5 \mathrm{mg}$ was statistically significantly superior to placebo at weeks 10 and 12 (ANOVA, $P<0.05$, observed cases) but not at week 12 (last observation carried forward). Paroxetine $20 \mathrm{mg}$ was statistically significantly superior to placebo at weeks 4,8 and 10 (ANOVA, $P<0.05$, observed cases). Escitalopram $10 \mathrm{mg}$ was significantly superior to paroxetine $20 \mathrm{mg}$ at week 12 (ANOVA, $P<0.05$, last observation carried forward).

\section{Response}

Response based on the $\geqslant 50 \%$ reduction in HAMA total score criterion was analysed for each treatment group at each visit by 


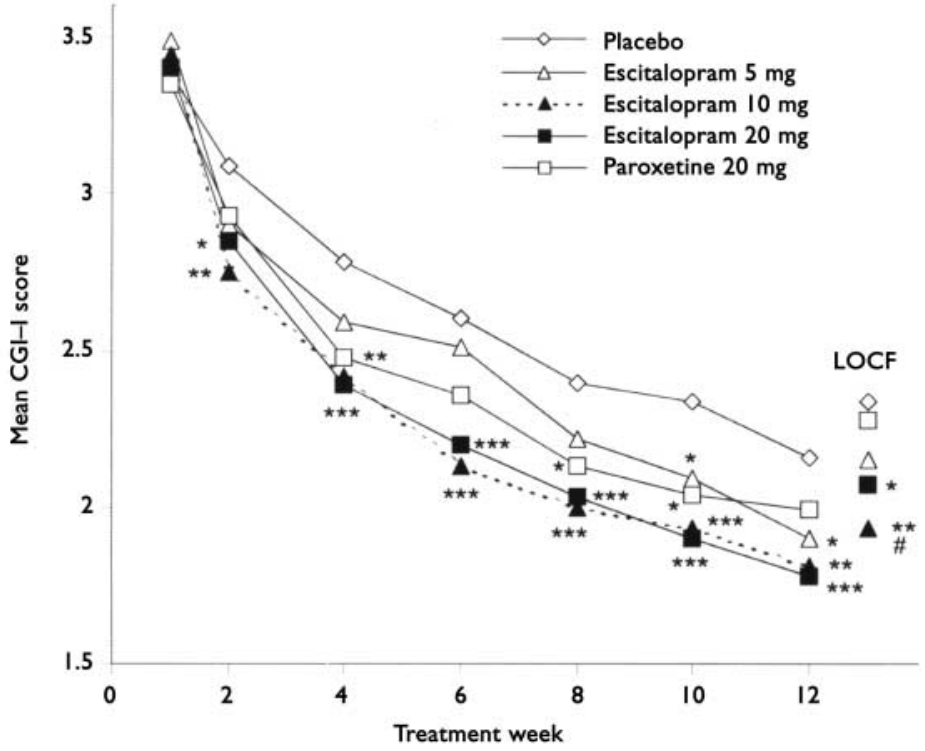

Fig. 3 Mean Clinical Global Impression - Improvement (CGI-I) score according to visit (intention to treat, observed cases) and at week 12, LOCF (last observation carried forward). Difference v. placebo, $* P<0.05$; $* * P<0.01$; ***P $<0.001$. Difference $v$. paroxetine, $\# P<0.05$ (analysis of covariance). observed cases and at week 12 by last observation carried forward (data not shown). Significant (Fisher's exact test, observed cases; $P<0.05)$ superiority in response $v$. placebo $(43 \%)$ was seen at week 6 for escitalopram $10 \mathrm{mg}$, and at weeks 10 and 12 (Fisher's exact test, $P<0.05$ ) for escitalopram $20 \mathrm{mg}$. Escitalopram $20 \mathrm{mg}$ was significantly $(80 \%$; Fisher's exact test, $P<0.05)$ superior to paroxetine $20 \mathrm{mg}$ carried-forward analysis, escitalopram $10 \mathrm{mg}$ was significantly (72\%; Fisher's exact test, $P<0.05)$ superior to paroxetine $20 \mathrm{mg}$ at week $12(60 \%)$. The observed cases response rate at week 12 for $(68 \%)$ at week 12 . In the last-observation- escitalopram $5 \mathrm{mg}$ (75\%) was not significantly different from placebo $(67 \%)$.

For response based on CGI-I, escitalopram $10 \mathrm{mg}$ was significantly (Fisher's exact test, $P<0.05$ ) superior to paroxetine $20 \mathrm{mg}$ at weeks 2 (last observation carried forward; $41 \%$ v. $29 \%$ respectively, data not shown) and $12(78 \% \quad v .66 \%$ respectively; Fig. 4). Significant superiority over placebo was seen from week 2 (observed cases) onwards for escitalopram $10 \mathrm{mg}$ (Fisher's exact test, $P<0.05$; Fig. 4). The escitalopram $20 \mathrm{mg}$ group showed significance (Fisher's exact test, $P<0.05$ ) over placebo from week 4 onwards. Response rates (observed cases) at

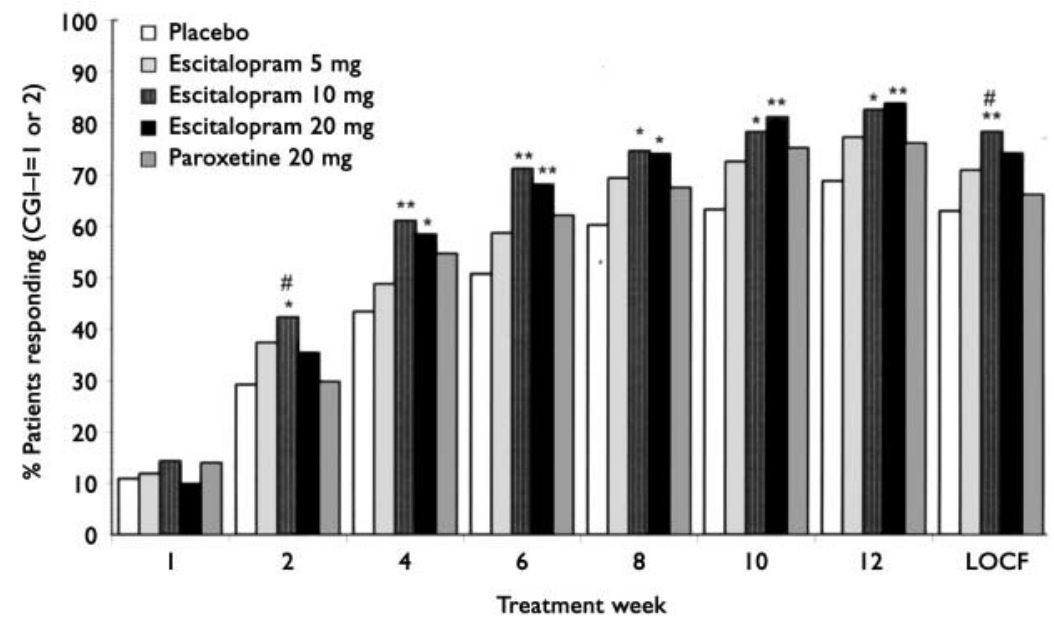

Fig. 4 Proportion of patients responding (defined as Clinical Global Impression - Improvement (CGI-I) score of I or 2) according to visit (intention to treat, observed cases) and at week 12, LOCF (last observation carried forward). Difference $v$. placebo, $* P<0.05$; $* P<0.01$. Difference $v$. paroxetine, $\# P<0.05$ (Fisher's exact test). week 12 were $69 \%$ (placebo), $77 \%$ (escitalopram $5 \mathrm{mg}$ ), $83 \%$ (escitalopram $10 \mathrm{mg}$ ), $84 \%$ (escitalopram $20 \mathrm{mg}$ ), and $76 \%$ (paroxetine $20 \mathrm{mg}$ ). The proportion of responders in the paroxetine $20 \mathrm{mg}$ group was not significantly different from that in the placebo group at any visit.

\section{Remission}

Remission was prospectively defined as a HAMA total score $\leqslant 7$ and was analysed for each treatment group at each visit by observed cases and at week 12 also by last observation carried forward. Superiority of escitalopram over placebo at week 12 was seen for all three doses of escitalopram (Fisher's exact test, $P<0.05$ ), and escitalopram $10 \mathrm{mg}$ was significantly $(48 \%$; Fisher's exact test, $P<0.05)$ superior to paroxetine $20 \mathrm{mg}$ (33\%). Superiority over placebo was seen at week 12 for escitalopram $5 \mathrm{mg}$ and from week 8 onwards for escitalopram 10 and $20 \mathrm{mg}$ (Fisher's exact test, observed cases, $P<0.05$; Fig. 5).

\section{HAD anxiety sub-scale scores}

In the analyses of the HAD anxiety subscale score, separation from placebo was statistically significant at both assessments (weeks 6 and 12) for escitalopram 10 and $20 \mathrm{mg}$ (ANCOVA, $P<0.05$ ), whereas escitalopram $5 \mathrm{mg}$ and paroxetine $20 \mathrm{mg}$ were significantly superior to placebo only at week 6 (ANCOVA, $P<0.05$; Fig. 6). In the last-observation-carried-forward analysis, escitalopram $10 \mathrm{mg}$ was furthermore significantly (ANCOVA, $P<0.05$ ) superior to paroxetine $20 \mathrm{mg}$ at week 12 .

\section{Tolerability}

Adverse events

Table 4 shows adverse events with an incidence $\geqslant 5 \%$ in any treatment group during the 12-week double-blind treatment period; there was no statistically significant difference in the number of patients experiencing adverse events across groups (chisquare test). The investigators considered the majority of the adverse events in all treatment groups to be mild or moderate. The approximate percentage of patients with adverse events considered to be related to study medication in each group was: $36 \%$ for placebo; $44 \%$ for escitalopram $5 \mathrm{mg} ; 54 \%$ for escitalopram $10 \mathrm{mg} ; 53 \%$ 


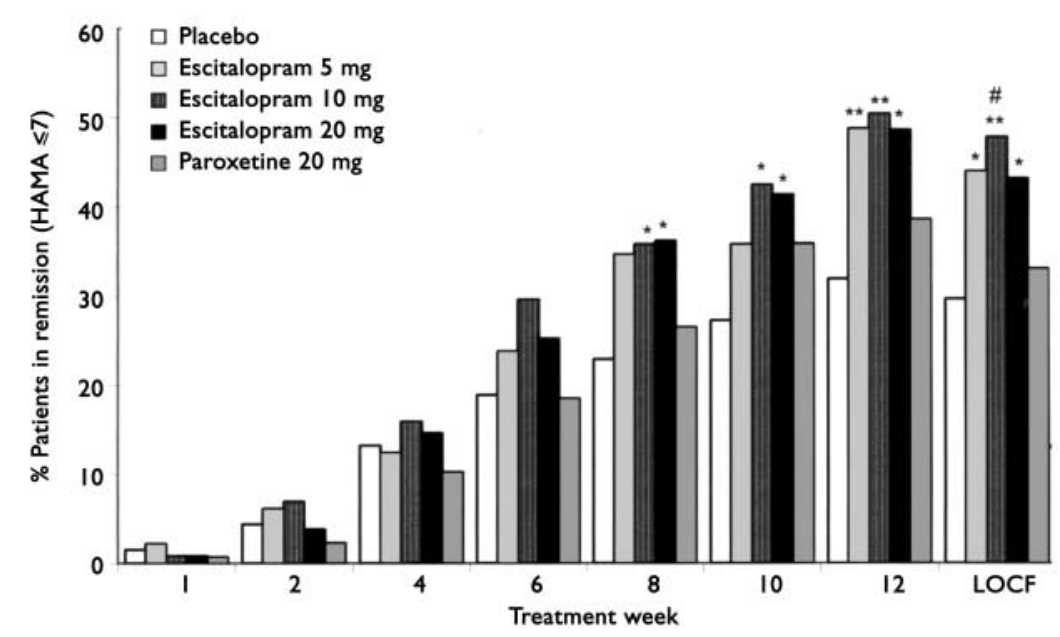

Fig. 5 Proportion of patients in remission (defined as a HAMA total score $\leqslant 7$ ) according to visit (intentionto-treat, observed cases) and at week 12, LOCF (last observation carried forward). Difference v. placebo, $* P<0.05 ; * * P<0.01$. Difference $v$. paroxetine, $\# P<0.05$ (Fisher's exact test).

for escitalopram $20 \mathrm{mg}$; and $55 \%$ for paroxetine $20 \mathrm{mg}$. The incidence of fatigue, insomnia, diarrhoea, somnolence, increased sweating, yawning and anorgasmia were statistically significantly higher in at least one treatment group $v$. placebo (Fisher's exact test).

Table 5 shows adverse events with an incidence $\geqslant 5 \%$ in any treatment group during the wash-out period. In each of the three escitalopram groups, the proportion of patients with adverse events during this period was not statistically different from with adverse events was significantly higher (chi-square test, $P<0.01$ ) than each of the events that had a statistically (Fisher's exact test, $P<0.05$ ) higher incidence in any active treatment group $v$. placebo during the insomnia (5.1\%); escitalopram $20 \mathrm{mg}$, vertigo $(3.6 \%)$; and paroxetine $20 \mathrm{mg}$, dizziness $(19.5 \%)$, nausea $(8.0 \%)$ and verti- that in the placebo group. The proportion of patients in the paroxetine $20 \mathrm{mg}$ group other active treatment groups. The adverse wash-out period were: escitalopram $10 \mathrm{mg}$, go $(5.3 \%)$. Dizziness had a statistically

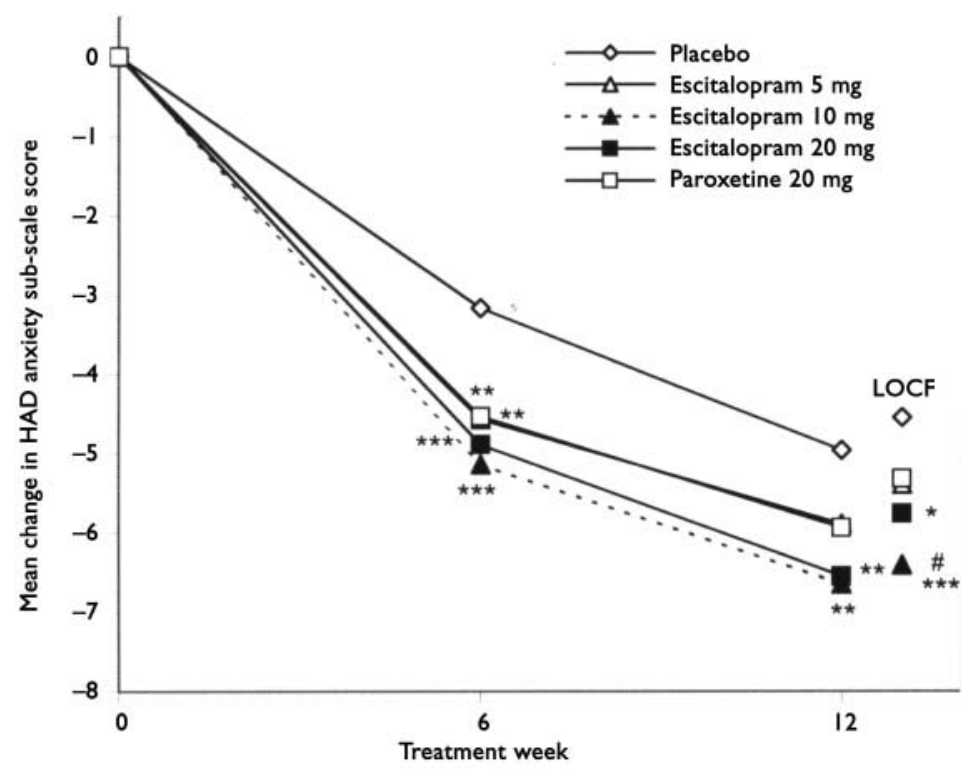

Fig. 6 Mean change from baseline in Hospital Anxiety and Depression (HAD) anxiety sub-scale score (intention-to-treat, observed cases) and at week 12, LOCF (last observation carried forward). Difference v. placebo, $* P<0.05$; ${ }^{* * P}<0.01$; ${ }^{* * * P}<0.001$. Difference $v$. paroxetine, ${ }^{*} \boldsymbol{P}<0.05$ (analysis of covariance). significantly higher incidence in the paroxetine $20 \mathrm{mg}$ group than in any of the escitalopram groups.

\section{Discontinuation Emergent Signs and Symptoms (DESS)}

Fig. 7 shows the adjusted mean change from the start of the wash-out period in the total score on the modified DESS, as assessed by the DESS checklist. The dose received by participants in the escitalopram $20 \mathrm{mg}$ group was down-tapered to $10 \mathrm{mg}$ during week 13 and to placebo during week 14, without a randomised withdrawal design, and results are therefore not presented in Fig. 7. The mean total scores on the modified DESS were at a maximum after 7 days of wash-out treatment for the paroxetine $20 \mathrm{mg}$ group and the 5 and $10 \mathrm{mg}$ escitalopram groups. The mean change in the number of new or worsened DESS items was statistically significantly higher in the paroxetine $20 \mathrm{mg}$ group than in the placebo group (4.2 v. 0.4; ANCOVA, $P<0.001)$ at day 7 . The discontinuation symptoms were transient and, after a further 7 days of wash-out treatment, returned to a level only slightly higher than that before starting wash-out treatment.

\section{DISCUSSION}

The aim of the current study was to examine three doses of escitalopram $v$. placebo and an active comparator (paroxetine) with proven efficacy for the medium-term (12 weeks) treatment of generalised anxiety disorder. The study design also allowed detailed evaluation of discontinuation symptoms, based on the DESS score. The baseline HAMA total score of approximately 27 and the baseline CGI-S score of approximately 4.5 indicate that this study population represents patients with moderate to severe illness.

There are a number of limitations to this study. First, the presence of the comorbid disorders typically found in patients with generalised anxiety disorder was low, as required by the protocol, and the results of this study are potentially less generalisable to samples seen in other clinical settings, although escitalopram has proven efficacy in major depression (Burke et al, 2002; Lepola et al, 2003), the most common comorbid disorder in generalised anxiety. Second, the placebo response rate $(>60 \%)$ is high, when compared with 
Table 4 Adverse events with an incidence of $\geqslant 5 \%$ according to group in the 12 -week double-blind treatment period

\begin{tabular}{|c|c|c|c|c|c|}
\hline Incidence of adverse events & PBO & ESC $5 \mathrm{mg}$ & $\mathrm{ESC} 10 \mathrm{mg}$ & ESC $20 \mathrm{mg}$ & PAR $20 \mathrm{mg}$ \\
\hline Patients treated, $n$ & 139 & 134 & 136 & 133 & 139 \\
\hline Patients with adverse events, $n$ (\%) & $88(63.3)$ & $88(65.7)$ & $94(69.1)$ & $94(70.7)$ & $101(72.7)$ \\
\hline Nausea & $17(12.2)$ & $20(14.9)$ & $28(20.6)$ & $28(2 I .1)$ & $30(21.6)$ \\
\hline Fatigue & $4(2.9)$ & II (8.2) & $14(10.3)^{*}$ & $22(16.5)^{*}$ & $12(8.6)$ \\
\hline Headache & $23(16.5)$ & $21(15.7)$ & $34(25.0)$ & $21(15.8)$ & $13(9.4)$ \\
\hline Insomnia & $3(2.2)$ & $12(9.0)^{*}$ & $17(12.5)^{*}$ & $14(10.5)^{*}$ & $15(10.8)^{*}$ \\
\hline Diarrhoea & $4(2.9)$ & $13(9.7)^{*}$ & $13(9.6)^{*}$ & 13 (9.8)* & II (7.9) \\
\hline Dizziness & $8(5.8)$ & $6(4.5)$ & $14(10.3)$ & $12(9.0)$ & $8(5.8)$ \\
\hline Rhinitis & $8(5.8)$ & $6(4.5)$ & $9(6.6)$ & $12(9.0)$ & $10(7.2)$ \\
\hline Sweating increased & $4(2.9)$ & $4(3.0)$ & II (8.I) & 12 (9.0)* & $12(8.6)$ \\
\hline Somnolence & $3(2.2)$ & $10(7.5)^{*}$ & $5(3.7)$ & $10 \quad(7.5)^{*}$ & $10(7.2)$ \\
\hline Ejaculation failure (men) & - & I (I.8) & $3(6.7)$ & $3(7.3)$ & $5(9.1)$ \\
\hline Mouth dry & $3(2.2)$ & $4(3.0)$ & $9(6.6)$ & $9(6.8)$ & $7(5.0)$ \\
\hline Libido decreased & $3(2.2)$ & $4(3.0)$ & $3(2.2)$ & $8(6.0)$ & $9(6.5)$ \\
\hline Yawning & - & I (0.7) & I $(0.7)$ & $7(5.3)^{*}$ & $3(2.2)$ \\
\hline Abdominal pain & $5(3.6)$ & $6(4.5)$ & $4(2.9)$ & $4(3.0)$ & $9(6.5)$ \\
\hline Anxiety & $4(2.9)$ & $9(6.7)$ & $3(2.2)$ & $4(3.0)$ & $6(4.3)$ \\
\hline Back pain & $4 \quad(2.9)$ & $4(3.0)$ & $7(5.1)$ & $4(3.0)$ & $5(3.6)$ \\
\hline Anorgasmia & - & $2(1.5)$ & $6(4.4)^{*}$ & $2(1.5)$ & $9(6.5)^{*}$ \\
\hline Ejaculation disorder (men) & - & - & - & - & $3(5.5)$ \\
\hline
\end{tabular}

ESC, escitalopram; PAR, paroxetine; PBO, placebo.

$P<0.05$ v. placebo (Fisher's exact test).

previous flexible-dose or fixed-dose SSRI acute treatment studies in anxiety disorder, which report placebo response rates of 37\% (flexible; Allgulander et al, 2004), $38 \%$ (flexible; Davidson et al, 2004), $46 \%$ (fixed; Rickels et al, 2003), 47\% (flexible; Pollack et al, 2001) and 54\% (flexible; Brawman-Mintzer et al, 2006). The high response rate to placebo in this investigation may result from the combination of the five-arm study design, the inclusion of multiple study centres and the frequency of study assessments. Third, there was no taper from $20 \mathrm{mg}$ to $10 \mathrm{mg}$ of paroxetine during the wash-out period, reflecting treatment recommendations at the time of the study. A fourth potential limitation lies within the method of analysis. It has been argued that last-observation-carried-forward analysis is not the best approach for evaluating data from randomised controlled trials (White et al, 2003; Everitt $\&$ Wessely, 2004). All methods for the imputation of missing data have their

Table 5 Adverse events with an incidence $\geqslant 5 \%$ according to group completing 12 weeks of treatment during the wash-out period

\begin{tabular}{|c|c|c|c|c|c|}
\hline Incidence of adverse events & PBO & ESC $5 \mathrm{mg}$ & ESC $10 \mathrm{mg}$ & ESC $20 \mathrm{mg}$ & PAR $20 \mathrm{mg}$ \\
\hline Completers, $n$ & 124 & 117 & 118 & III & 113 \\
\hline Patients with adverse events, $n(\%)$ & $24(19.4)$ & $13(11.1)$ & $30(25.4)$ & $21(18.9)$ & $47(4 I .6)^{*}$ \\
\hline Dizziness & $2(1.6)$ & I $(0.9)$ & $4(3.4)$ & $4(3.6)$ & $22(19.5)^{* \#}$ \\
\hline Headache & $8(6.5)$ & $3(2.6)$ & $6(5.1)$ & $4(3.6)$ & $6(5.3)$ \\
\hline Vertigo & & & I $(0.8)$ & 4 (3.6)* & $6(5.3)^{*}$ \\
\hline Insomnia & & & $6(5 . I)^{*}$ & $2(1.8)$ & I (0.9) \\
\hline Nausea & & & $4(3.4)$ & I (0.9) & $9(8.0)^{*}$ \\
\hline
\end{tabular}

ESC, escitalopram; PAR, paroxetine; PBO, placebo.

${ }^{*} P<0.05$ v. placebo; ${ }^{\# P}<0.05$ v. escitalopram (any dose) (Fisher's exact test).

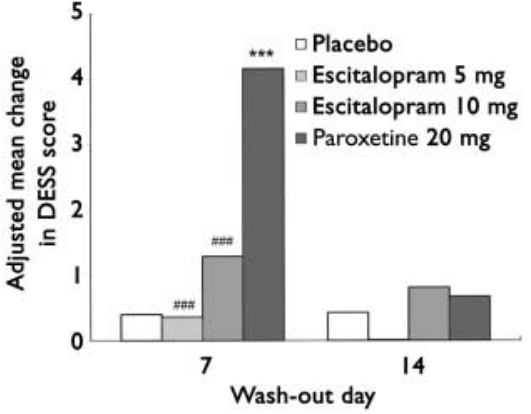

Fig. 7 Adjusted mean change from the start of the wash-out period in the modified Discontinuation Emergent Signs and Symptoms (DESS) total score (patients completed, observed cases). Difference v. placebo, $* * * P<0.001$; difference $v$. paroxetine $\# \# \#<0.001$ (analysis of covariance).

limitations, but in disorders that do not deteriorate progressively, the conservative approach adopted in the above analysis is favoured by regulatory bodies, and was the specified form of data analysis in the study protocol.

The primary efficacy analysis (mean change from baseline in HAMA total score at week 12 using last observation carried forward) showed that escitalopram 10 and $20 \mathrm{mg}$ were significantly superior to placebo. A dose-response relationship was seen at week 12 in the observed cases analysis. All three escitalopram doses were significantly superior to placebo; however, there was an increasing robustness of significance $v$. placebo from escitalopram $5 \mathrm{mg}$ to $20 \mathrm{mg}$, which was associated with the highest response. Escitalopram $20 \mathrm{mg}$ was also superior to paroxetine $20 \mathrm{mg}$ in the observed cases analysis. The last-observationcarried-forward analysis demonstrated that escitalopram $10 \mathrm{mg}$ was also significantly superior to paroxetine $20 \mathrm{mg}$ at week 12 . Mean HAMA and CGI-S scores decreased from week 10 to week 12 , indicating that continued treatment might have resulted in further improvement, as found in a relapseprevention study (Allgulander et al, 2005), although only responders to acute treatment were eligible to continue in that study.

Paroxetine, $20 \mathrm{mg}$ failed to show a significant difference from placebo in the primary efficacy analysis, which is probably attributable to the high response to placebo (Fig. 4). However, the decrease in HAMA from baseline to week 12 with paroxetine was approximately 14 points, numerically greater than placebo, and similar to that described in a previous study (Rickels $e t$ al, 2003), where paroxetine $20 \mathrm{mg}$ for 8 
weeks resulted in a 12.5-point reduction in the total HAMA score, and paroxetine $40 \mathrm{mg}$ in a reduction of 12.2 points. In our study, paroxetine appeared efficacious on some of the secondary outcome measures: for example, it was significantly superior to placebo on the CGI-I at several points.

There were a number of secondary outcome measures, including the CGI-I, response and remission rates and the HAD anxiety sub-scale score. Using the CGI-I, in the observed cases analysis, the escitalopram 10 and $20 \mathrm{mg}$ doses were superior to placebo from week 2 onwards, as well as at week 12 using last observation carried forward. According to response criteria, escitalopram $10 \mathrm{mg}$ was superior to placebo from week 2 onwards and, at the end of the study, escitalopram 10 and $20 \mathrm{mg}$ doses were significantly more efficacious than placebo. In the last-observation-carried-forward analysis, the response rate for the escitalopram $10 \mathrm{mg}$ was also superior to paroxetine $20 \mathrm{mg}$. According to remission criteria, escitalopram 10 and $20 \mathrm{mg}$ doses were more efficacious than placebo from week 8 onwards. Paroxetine $20 \mathrm{mg}$ did not separate from placebo at any time point in this analysis. At study end, all three doses of escitalopram were superior to placebo and, in the last observation analysis, escitalopram $10 \mathrm{mg}$ was superior to paroxetine $20 \mathrm{mg}$.

For the self-rating HAD anxiety subscale score, escitalopram 10 and $20 \mathrm{mg}$ were significantly better than placebo at weeks 6 and 12. In the last-observationcarried-forward analysis, at 12 weeks escitalopram $10 \mathrm{mg}$ was also superior to paroxetine $20 \mathrm{mg}$. This analysis showed a close agreement between the investigator's and the patient's assessment of treatment outcome.

Escitalopram $5 \mathrm{mg}$ was not significantly superior to placebo across a variety of primary and secondary measures. This indicates that escitalopram $5 \mathrm{mg}$ is probably too low a dose in this population of patients with generalised anxiety disorder. Higher doses are more efficacious, with an increased benefit for $20 \mathrm{mg}$, especially in terms of reaching symptomatic remission.

The incidence of adverse events during the 12-week study period was similar across all treatment groups. The proportion of patients with adverse events and withdrawals due to adverse events tended to increase as the dose of escitalopram increased. Only in the highest escitalopram

DAVID S. BALDWIN, DM, Clinical Neuroscience Division, School of Medicine, University of Southampton, UK; ANNA KARINA TRAP HUUSOM, MSc, H. Lundbeck A/S, Copenhagen, Denmark; ELI MAHLUM, MSc, H. Lundbeck A/S, Lysaker, Norway

Correspondence: David S. Baldwin, University Department of Mental Health, Royal South Hants Hospital, Graham Road, Southampton SOI4 OYG, UK. Tel. : +44 (0) 2380825533 ,

fax: +44 (0) 2380234243 , email: dsbl @soton.ac.uk

(First received 29 April 2005, final revision 23 February 2006, accepted 2 March 2006)

dose group was there a significant difference in withdrawals due to adverse events compared with withdrawals with the placebo group, as was the case in the paroxetine $20 \mathrm{mg}$ group. The adverse events that were reported during the 12 -week treatment period for both escitalopram and paroxetine were characteristic for SSRIs. The incidence of patients reporting such events during the wash-out period was significantly higher in the paroxetine $20 \mathrm{mg}$ group compared with escitalopram and placebo. The most frequent adverse event during wash-out was dizziness, which was reported by almost one-fifth of patients (22 out of 113) in the paroxetine group.

After 7 days of wash-out treatment, patients in the paroxetine $20 \mathrm{mg}$ group were significantly more likely to have discontinuation-emergent symptoms compared with those in the placebo group. Patients who stopped treatment with escitalopram $10 \mathrm{mg}$ had fewer discontinuation effects than those who stopped treatment with paroxetine $20 \mathrm{mg}$, this being consistent with the findings of a similar placebo-controlled study in social phobia (Lader et al, 2004). The discontinuation symptoms in the DESS checklist were transient and (after a further 7 days of wash-out treatment with placebo) returned to a level similar to that before patients started wash-out treatment. There was no significant difference between the escitalopram $5 \mathrm{mg}$ and $10 \mathrm{mg}$ groups $v$. placebo based on the modified DESS total score. In view of this, and the lower number of patients withdrawn because of adverse events in the escitalopram $10 \mathrm{mg}$ group, clinicians may prefer to start with a $10 \mathrm{mg}$ dose, increasing to $20 \mathrm{mg}$ if patients show no signs of response after 4 weeks of treatment, when a significant difference from response to placebo was first seen in this study (Fig. 2).

In summary, escitalopram (10 and $20 \mathrm{mg} /$ day) was efficacious and well tolerated in the medium-term treatment of generalised anxiety disorder. Escitalopram
$10 \mathrm{mg}$ was significantly more efficacious than paroxetine $20 \mathrm{mg}$.

\section{REFERENCES}

Allgulander, C., Dahl, A., Austin, C., et al (2004) Efficacy of sertraline in a 12-week trial for generalized anxiety disorder. American Journal of Psychiatry, 161, 1642-1649.

Allgulander, C., Florea, I. \& Huusom, A. K.T. (2005) Prevention of relapse in generalised anxiety disorder by escitalopram treatment. International journal of Neuropsychopharmacology, 2005. doi: 10.1017/ SI461145705005973

American Psychiatric Association (2000) Diagnostic and Statistical Manual of Mental Disorders (4th edn, text revision) (DSM-IV-TR). Washigton, DC: APA.

Baldwin, D. S. \& Polkinghorn, C. (2005) Evidencebased pharmacological treatment of generalised anxiety disorder. International journal of Neuropsychopharmacology, 8, 293-302.

Bielski, R. J., Bose, A. \& Chang, C. C. (2005) A double-blind comparison of escitalopram and paroxetine in the long-term treatment of generalized anxiety disorder. Annals of Clinical Psychiatry, 17, 65-69.

Brawman-Mintzer, O., Knapp, R. G., Rynn, M., et al (2006) Sertraline treatment for generalized anxiety disorder: a randomized, double-blind, placebocontrolled study. Journal of Clinical Psychiatry, 67, 874-881.

Burke, W. J., Gergel, I. \& Bose, A. (2002) Fixed-dose trial of the single isomer SSRI escitalopram in depressed outpatients. Journal of Clinical Psychiatry, 63, 33I-336.

Davidson, J. R.T., Bose, A., Korotzer, A., et al (2004) Escitalopram in the treatment of generalized anxiety disorder: a double-blind, placebo controlled, flexible dose study. Depression and Anxiety, 19, 234-240.

Everitt, B. S. \& Wessely, S. (2004) Clinical Trials in Psychiatry. Oxford: Oxford University Press.

Goodman, W. K., Bose, A. \& Wang, Q. (2005) Treatment of generalized anxiety disorder with escitalopram: pooled results from double-blind, placebo-controlled trials. Journal of Affective Disorders, 87, $|6|-167$.

Guy, w. (1976) ECDEU Assessment Manual for Psychopharmacology. US Department of Health, Education, and Welfare. DHEW Publication No. (ADM) 218-222. Kensington, MD: George Washington University.

Hamilton, M. (1959) The assessment of anxiety states by rating. British Journal of Medical Psychology, 32, 50-55.

ICH (1996) ICH Harmonised Tripartite Guideline E6: Guideline for Good Clinical Practice. http: / /www.ich.org/ LOB/media/MEDIA482.pdf 
Katz, I. R., Reynolds, III, C. F., Alexopoulos, G. S., et al (2002) Venlafaxine ER as a treatment for generalized anxiety disorder in older adults: pooled analysis of five randomized placebo-controlled clinical trials. Journal of the American Geriatric Society, 50, 18-25.

Kessler, R. C., Keller, M. B. \& Wittchen, H. U. (200I)

The epidemiology of generalised anxiety disorder.

Psychiatric Clinics of North America, 24, 19-39.

Lader, M., Stender, K., Bürger, V., et al (2004) Efficacy and tolerability of escitalopram in 12- and 24-week treatment of social anxiety disorder: randomised, double-blind, placebo controlled, fixed-dose study Depression and Anxiety, 19, 24I-248.

\section{Lepola, U. M., Loft, H. \& Reines, E. H. (2003)} Escitalopram (10-20 mg/day) is effective and well tolerated in a placebo-controlled study in depression in primary care. International Clinical Psychopharmacology, 18, 211-217.
Montgomery, S. \& van Zwieten-Boot, B. (2002) ECNP Consensus Meeting March 2000 Guidelines for Investigating Efficacy in GAD. European Neuropsychopharmacology, I2, 81-87.

Montgomery, S. A. \& Åsberg, M. (1979) A new depression scale designed to be sensitive to change. British Journal of Psychiatry, 134, 382-389.

Pollack, M. H., Zaninelli, R., Goddard, A., et al (200I) Paroxetine in the treatment of generalised anxiety disorder: results of a placebo-controlled, flexible-dosage trial. Journal of Clinical Psychiatry, 62 350-358.

Rickels, K., Zaninelli, R., McCafferty, J., et al (2003) Paroxetine treatment of generalised anxiety disorder; a double-blind, placebo-controlled study. American Journal of Psychiatry, I60, 749-756.

Rosenbaum, J. F., Fava, M., Hoog, S. L., et al (1998) Selective serotonin reuptake inhibitor discontinuation syndrome: a randomized clinical trial. Biological Psychiatry, 44, 77-87.

Sheehan, D. V., Lecrubier, Y., Sheehan, K. H., et al (1998) The Mini International Neuropsychiatric Interview (M.I.N.I.): the development and validation of a structured diagnostic psychiatric interview for DSM-IV and ICD-10. Journal of Clinical Psychiatry, 59, 22-33.

White, I. R., Moodie, E., Thompson, S. G., et al (2003) A modelling strategy for the analysis of clinical trials with partly missing longitudinal data. International journal of Methods in Psychiatric Research, 12, 139-150.

World Medical Association (2000) Declaration of Helsinki: Ethical Principles for Medical Research Involving Human Subjects. http: / /www.wma.net/e/policy/ b3.htm

Zigmond, A. S. \& Snaith, R. P. (1983) The Hospital Anxiety and Depression scale. Acta Psychiatrica Scandinavica, 67, 361-370. 